ISSN 1991-8631

Original Paper

http://indexmedicus.afro.who.int

\title{
Contribution des fourmis à la lutte biologique contre les Loranthaceae
}

\author{
C. F. LADOH YEMEDA ${ }^{1 *}$, Ruth MONY ${ }^{2}$, Mathurin TCHATAT $^{3}$ et \\ Siegfried DIBONG ${ }^{1,4,5}$ \\ ${ }^{I}$ Département de Biologie des Organismes Végétaux, Faculté des Sciences, Université de Douala, \\ B.P. 24157 Douala, Cameroun. \\ ${ }^{2}$ Département de Biologie et Physiologie Animales, Faculté des Sciences, Université de Yaoundé 1, B.P. 812 \\ Yaoundé,Cameroun. \\ ${ }^{3}$ IRAD Nkolbisson, B.P. 2067, Yaoundé, Cameroun. \\ ${ }^{4}$ Département des Sciences Pharmaceutiques, Faculté de Médecine et des Sciences Pharmaceutiques, \\ Université de Douala, B.P. 2701 Douala, Cameroun. \\ ${ }^{5}$ Institut des Sciences Halieutiques, Université de Douala, B.P. 2701 Douala, Cameroun. \\ *Auteur correspondant, E-mail : christieflora@yahoo.fr
}

\section{RESUME}

Les Loranthaceae sont des plantes parasites qui causent de nombreux dommages sur les plantes hôtes se traduisant par une réduction de la floraison, de la fructification et de la production. Dans le but de trouver une méthode de lutte efficace contre les Loranthaceae, une étude relative à l'activité myrmécophile a été menée au verger de la chefferie de Ndogbong. Un inventaire de la myrmécofaune a été réalisé sur 16 individus de Theobroma cacao dont 9 parasités et 7 non parasités. Trois espèces de Loranthaceae ont été recensées: Phragmanthera batangae (Engler) S. Balle, P. capitata (Sprengel) S. Balle et Tapinanthus preussii (Engler) Van Teghem. Trois sous-familles de fourmis ont été identifiées : les Ponerinae (Odontomachus troglodytes Santschi), les Formicinae (Camponotus sp.) et les Myrmicinae (Crematogaster sp., C. decacrema, C. oxygyne, et Pheidole megacephala Fabricius). Deux espèces de fourmis ont été présentes sur les fleurs et les fruits de $P$. capitata et $T$. preussii et contribuent à la lutte biologique contre les Loranthaceae.

(C) 2013 International Formulae Group. All rights reserved.

Mots clés: Myrmécofaune, lutte, Loranthaceae, Ndogbong, Theobroma cacao.

\section{INTRODUCTION}

Les Loranthaceae sont des phanérogames hémiparasites épiphytes qui, implantées sur les parties aériennes de leurs hôtes sont responsables des dégâts économiques, écologiques, morphologiques variables selon les essences ligneuses parasitées (Sallé et al., 1998). Polhill et Wiens (1998) dénombrent plus de 500 espèces en
Afrique et en Arabie tandis que Balle (1986) en dénombre près de 26 identifiées au Cameroun et réparties dans 7 genres. Le parasitisme des Loranthaceae est un facteur néfaste pour la culture de nombreuses essences utiles telles que le cacao, le café, l'hévéa, les agrumes, les avocatiers au même titre que les nombreux ravageurs et maladies fongiques déjà étudiés (Sallé et al., 1998 ; 
Sonké et al., 2000). La perturbation la plus apparente causée par les Loranthaceae est le dépérissement de la partie distale des branches parasitées (Boussim et al., 1993). Le parasite détourne à son profit la sève brute destinée à l'extrémité distale de la branche parasitée. Ce déficit a pour conséquence l'arrêt de la croissance, le dépérissement puis la mort de l'hôte parasité (Boussim et al., 1993). Les fourmis jouent un rôle important dans le fonctionnement des écosystèmes. Classiquement dans les relations plantesfourmis, les fourmis reçoivent de la plante un abri ou de la nourriture, tandis que la plante est protégée par les fourmis contre des herbivores ou même contre d'autres plantes (Beattie et Hughes, 2002; Heil et Mckey, 2003). Les plantes myrmécophiles se trouvent sur tous les continents, surtout dans les tropiques et colonisent de nombreux groupes végétaux. Ainsi, les interactions plantesfourmis varient des associations facultatives diffuses aux associations spécifiques obligatoires (Dejean et al., 2007; Passera et Aron, 2005). Certaines fourmis prédatrices peuvent être de bons agents de lutte biologique contre des espèces phytophages, tandis que d'autres peuvent avoir un effet néfaste en agronomie en exploitant des Hémiptères nuisibles pour les plantes cultivées.

La présente étude a été entreprise au verger de la chefferie de Ndogbong, dans le but de rechercher l'impact de la myrmécophilie des plantes-hôtes sur la floraison et la fructification des Loranthaceae. Les objectifs spécifiques ont consisté à : (1) réaliser l'inventaire de toutes les espèces ligneuses du verger, (2) identifier et compter toutes les touffes de Loranthaceae et (3) suivre l'activité fourragère de la myrmécofaune pendant la floraison et la fructification des Loranthaceae afin d'en dégager l'incidence.

\section{MATERIEL ET METHODES Site d'étude}

L'étude a été réalisée dans un verger d'une superficie de 140 m x 200 m (28.000 $\mathrm{m}^{2}$ ) localisé dans le campement de la chefferie de Ndogbong, quartier situé au Nord-Est de la ville de Douala (0340'04 ${ }^{\circ} 11^{\prime} \mathrm{N}$ de latitude, $09^{\circ} 16^{\prime}-09^{\circ} 52^{\prime} \mathrm{E}$ de longitude et $13 \mathrm{~m}$ d'altitude) (Figure 1). La ville a un climat qui appartient au domaine équatorial dit camerounien qui se caractérise par deux saisons avec une longue saison des pluies (au moins 9 mois), des précipitations abondantes (environ $4000 \mathrm{~mm}$ par an), des températures élevées $\left(26,7{ }^{\circ} \mathrm{C}\right)$ et stables. La moyenne minimale de température à Douala pour 30 années (1961-1990) est de $22,6{ }^{\circ} \mathrm{C}$ en juillet et la moyenne maximale de température est de $32,3{ }^{\circ} \mathrm{C}$ en février. L'humidité relative de l'air reste élevée toute l'année et voisine de $100 \%$ (Din et al., 2008). Le verger du campement constitué de nombreux arbustes fruitiers, destinés à la consommation des propriétaires est une ancienne cacaoyère abandonnée.

\section{Méthodes d'étude Inventaire floristique}

L'étude a été réalisée sur une période allant de novembre 2010 à février 2011. Elle a consisté à inventorier tous les arbres (diamètre $\geq 5 \mathrm{~cm}$ ) du verger de la chefferie parasités ou non par les Loranthaceae. Pour chaque arbre, le nombre de touffes de Loranthaceae est compté, en grimpant sur l'arbre à l'échelle.

\section{Récoltes et identification des fourmis sur les individus}

Les fourmis ont été récoltées sur les individus de Theobroma cacao et sur les Loranthaceae entre $9 \mathrm{~h}$ et $11 \mathrm{~h}$ chaque samedi pendant huit semaines. Les fourmis ont été capturées à l'aide d'un aspirateur à bouche (cas des ouvrières de taille moyenne) ou d'une paire de pinces molles pour les ouvrières de plus grande taille et conservées dans des boîtes étiquetées contenant de l'éthanol à 70 ${ }^{\circ} \mathrm{C}$.

L'identification s'est faite au laboratoire avec l'aide des clés d'identification de la base des données des fourmis africaines (www.antbase.org) et leur nomenclature approuvée par les entomologistes du département de Biologie 
des Organismes Animaux de la Faculté des Sciences de l'Université de Douala.

L'activité des ouvrières a été suivie sur les fleurs de trois nœuds successifs d'un rameau de chaque touffe de Loranthaceae. A cet effet, les ouvrières ont été récoltées pendant leur période de fourragement dans le but d'évaluer leur abondance.

\section{Analyses des données}

Les données collectées ont été traitées par le logiciel Statview 5.0.

Les variables quantitatives ont été comparées par les tests de Wilcoxon et ANOVA. Le coefficient statistique de significativité ( $\mathrm{p}$-value) $<0,05$ signifie une différence statistique significative.

L'indice de Shannon-Weaver (H') a permis de déterminer la diversité des espèces ligneuses du milieu en prenant en compte le nombre d'espèces et l'abondance des individus au sein de chacune des espèces (Gray et al., 1992), elle est donnée par la relation :
$H=-\sum_{i=1}^{5} p_{i} \ln p_{i}$

Où

$P i=$ abondance relative des espèces: $P i=N_{i} / N$;

$S=$ nombre total d'espèces ;

$N_{i}=$ nombre d'individus d'une espèce dans le milieu ;

$N=$ nombre total d'individus de toutes les espèces dans le milieu.

Le taux de parasitisme (Pp) permettant d'évaluer le niveau d'infestation des Loranthaceae dans le verger de la chefferie a été calculé par la formule suivante :

Pp $=$ (nombre d'arbres parasités/nombre total d'arbre) x 100

Les courbes de régression montrent la corrélation qui existe entre floraison, fructification et fourmis. Le coefficient de détermination ou degré de corrélation $\left(\mathrm{R}^{2}\right)$ varie entre 0 et 1,0 étant une absence totale de corrélation et 1 une parfaite corrélation.

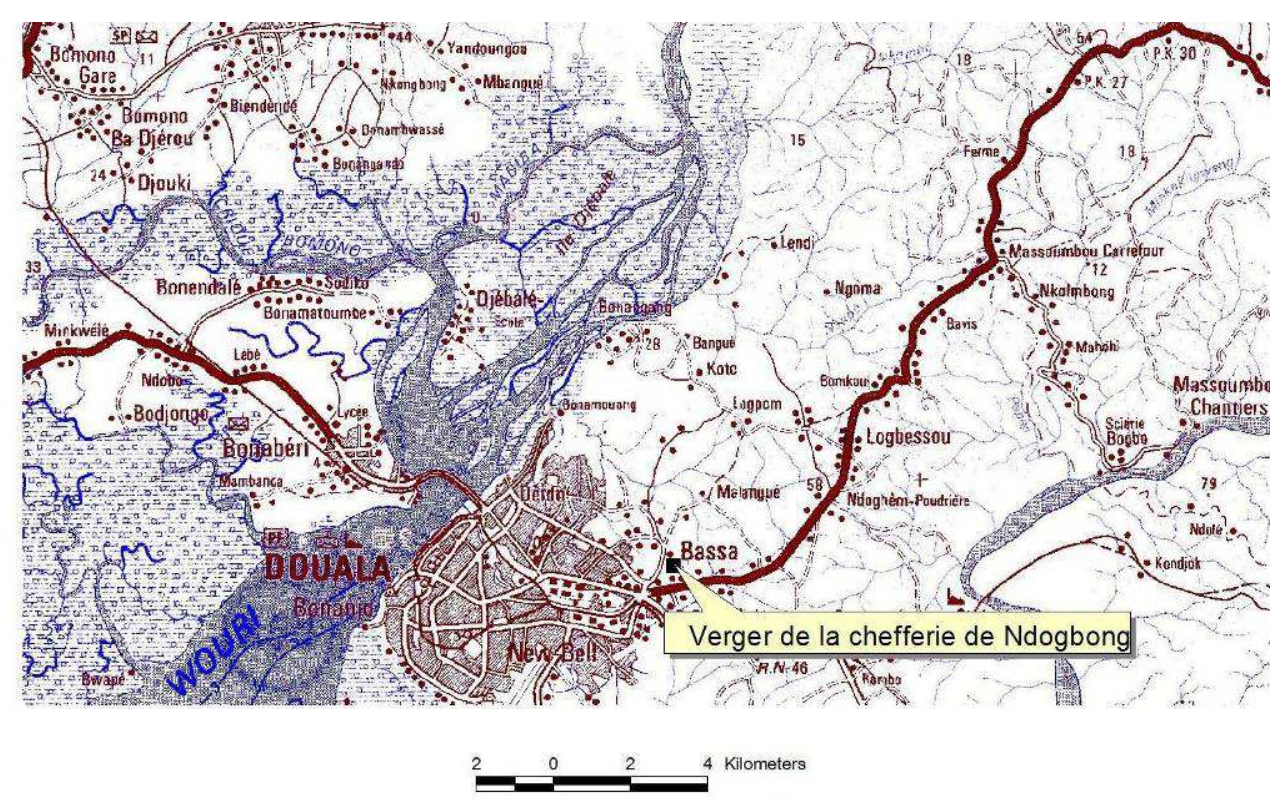

Figure 1 : Localisation du Campement de la Chefferie de Ndogbong (Logiciel Arcview GIS 3.2a). 


\section{RESULTATS}

\section{Inventaire floristique}

Au total, 49 arbustes appartenant à 11 espèces dont 9 genres et 8 familles ont été inventoriés. Les familles des Anarcadiaceae et des Sterculiaceae sont les mieux représentées avec 29 individus soit 59,2\% de la flore recensée. Toutes ces espèces sont exotiques (Citrus maxima Obsbeck, $C$. reticulata Blanco, $C$. sinensis Arancio, Dacryodes edulis (G. Don) H. J. Lam, Garcinia cola Heckel, Mangifera indica L., Manniophyton fulvum Müll.-Arg., Persea americana Mill., Psidium guajava L. et Theobroma cacao L.) excepté Spondias mangifera Willd., qui est autochtone et originaire de la forêt dense humide sempervirente littorale. Les espèces les plus abondantes sont Theobroma cacao L., Mangifera indica L. et Citrus spp. avec respectivement 16,13 et 10 individus soit 39 individus $(79,6 \%$ des arbustes recensés). $20,4 \%$ représente les espèces les moins abondantes avec 3 individus chacune (Persea americana Mill. et Psidium guajava L.) et les espèces peu abondantes avec un seul individu (Dacryodes edulis (G. Don) H. J. Lam., Garcinia cola Heckel., Manniophyton fulvum Mull.-Arg. et Spondias mangifera Willd.). L'indice de Shannon Weaver $\left(\mathrm{H}^{\prime}\right)$ est égal à 1,8 .

Trois espèces de Loranthaceae ont été recensées sur les individus de Theobroma cacao. Ce sont Phragmanthera batangae (Engler) S. Balle, P. capitata (Sprengel) S. Balle et Tapinanthus preussii (Engler) Van Teghem. Mais seule $P$. capitata a été abondante et fréquente sur les individus de $T$. cacao parasité. Le taux de parasitisme est de $59,18 \%$.

\section{Inventaire de la myrmécofaune}

$\mathrm{Au}$ total, 7 espèces de fourmis appartenant à 3 sous-familles ont été recensées sur les individus de Theobroma cacao :

- Les Ponerinae avec une seule espèce, Odontomachus troglodytes Santschi (Figure 2) ;

- Les Formicinae avec une seule espèce, Camponotus sp. (Figure 3) ;

- Les Myrmicinae ont été la sous-famille la plus diversifiée avec 4 espèces (Crematogaster sp., C. decacrema (Figure 4), $C$. oxygyne et Pheidole megacephala Fabricius). Parmi ces sous-familles, seule celle des Myrmicinae possède des espèces arboricoles.

Parmi les espèces de Loranthaceae parasitant les individus de Theobroma cacao, $P$. capitata et $T$. preussii étaient en fleurs permettant le suivi de l'activité fourragère des fourmis. Pendant toutes les périodes d'observation, deux espèces de fourmis Crematogaster decacrema et $C$. oxygyne ont été présentes sur $P$. capitata et une seule espèce, $C$. oxygyne sur $T$. preussii.

\section{Cas de Phragmanthera capitata}

Au total, 496 fourmis ont été récoltées sur les nœuds marqués de Phragmanthera capitata pendant 8 semaines, soient 200 fourmis/328 fleurs (Test de Wilcoxon, $\mathrm{p}=0,0431$ ) pendant la floraison et 296 fourmis/158 fruits (Test de Wilcoxon, $\mathrm{p}=0,0679$ ) pendant la fructification. L'histogramme de variation des fourmis montre que l'activité fourragère des fourmis pendant la floraison de $P$. capitata est plus élevée que pendant la fructification. Nonobstant la chute des fleurs, le nombre de fourmis sur les nœuds était moins important que pendant la floraison. Pendant la floraison et la fructification, le nombre de fourmis tout 
comme celui des fleurs et des fruits varient dans le même sens (Figure 5).

\section{Cas de Tapinanthus preussii}

Au total, 1410 fourmis ont été récoltées sur 278 fleurs des nœuds marqués de Tapinanthus preussii pendant une période de 8 semaines (Test de ANOVA, $\mathrm{p}=0,0114$ ). Aucune apparition de fruits n'a été constatée. L'histogramme de variation des fourmis montre que l'activité fourragère décroissait dans le même sens que le nombre de fleurs (Figure 6). Le nombre de fourmis sur les nœuds de $T$. preussii était plus élevé que sur les nœuds de Phragmanthera capitata bien que la floraison soit plus importante chez cette dernière. L'activité fourragère des fourmis ne se limitait pas seulement au niveau des fleurs et des fruits, mais également au niveau des feuilles et des suçoirs (Figure 10).

\section{Corrélation entre les fleurs, fruits des Loranthaceae et fourmis}

Les coefficients de corrélation pendant la floraison et la fructification significativement proches de 1 (Figures 7, 8 et 9), montrent une contribution directe des fourmis à la destruction des fleurs et des fruits des Loranthaceae. Le nombre de fleurs et de fruits est proportionnel au nombre de fourmis sur les nœuds des Loranthaceae: Plus il y a des fleurs et des fruits, plus il y a des fourmis sur les nœuds et inversement.

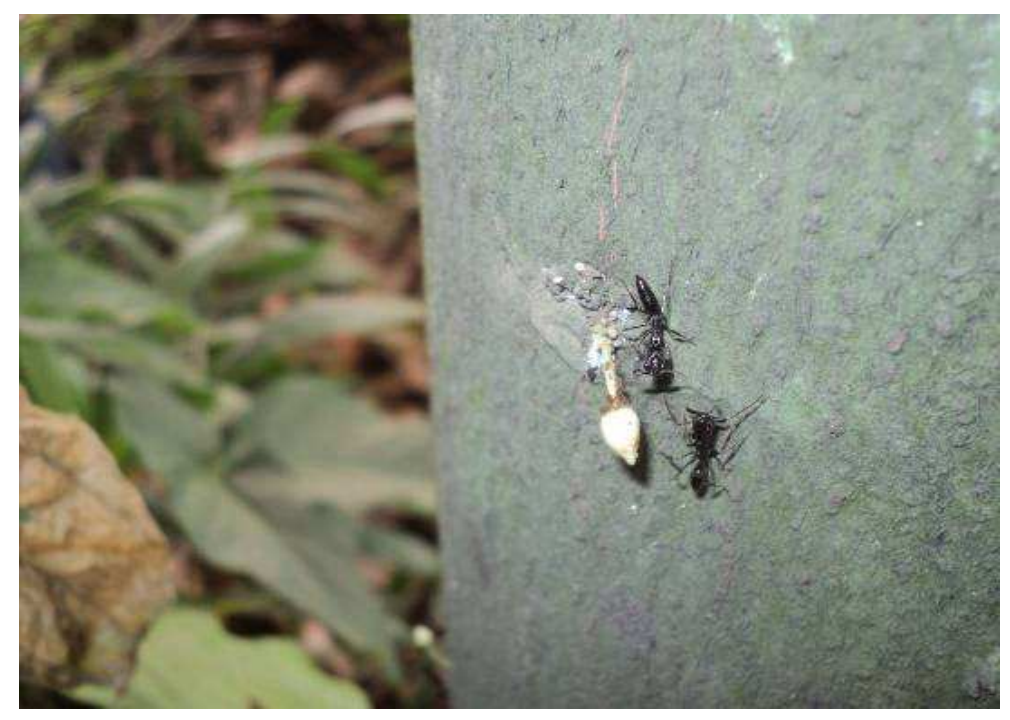

Figure 2 : Ouvrières de Odontomachus troglodytes Santschi. 


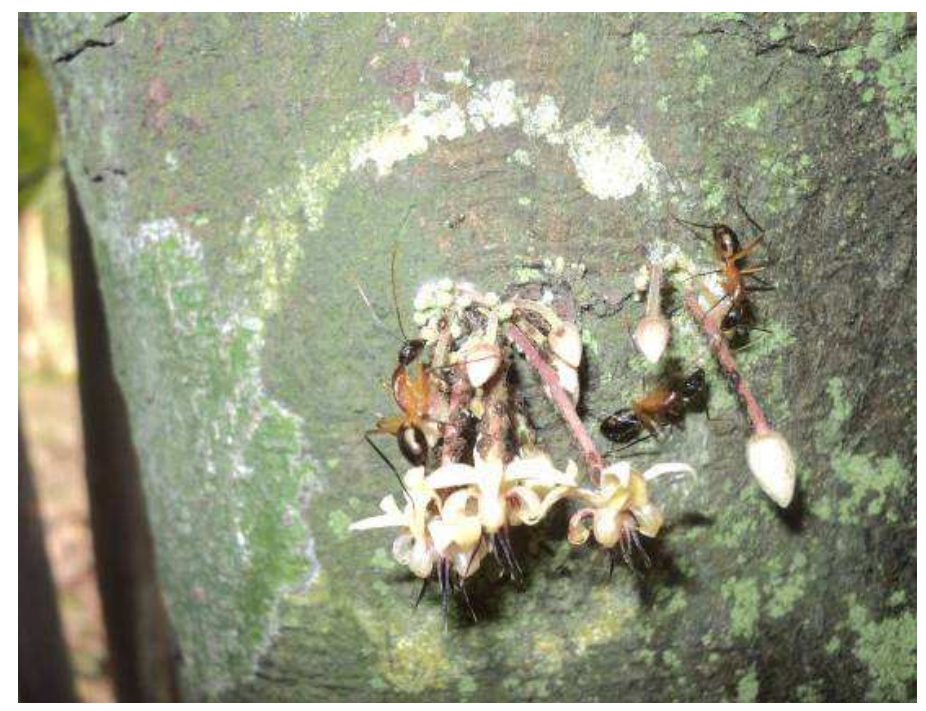

Figure 3 : Ouvrières de Camponotus sp.

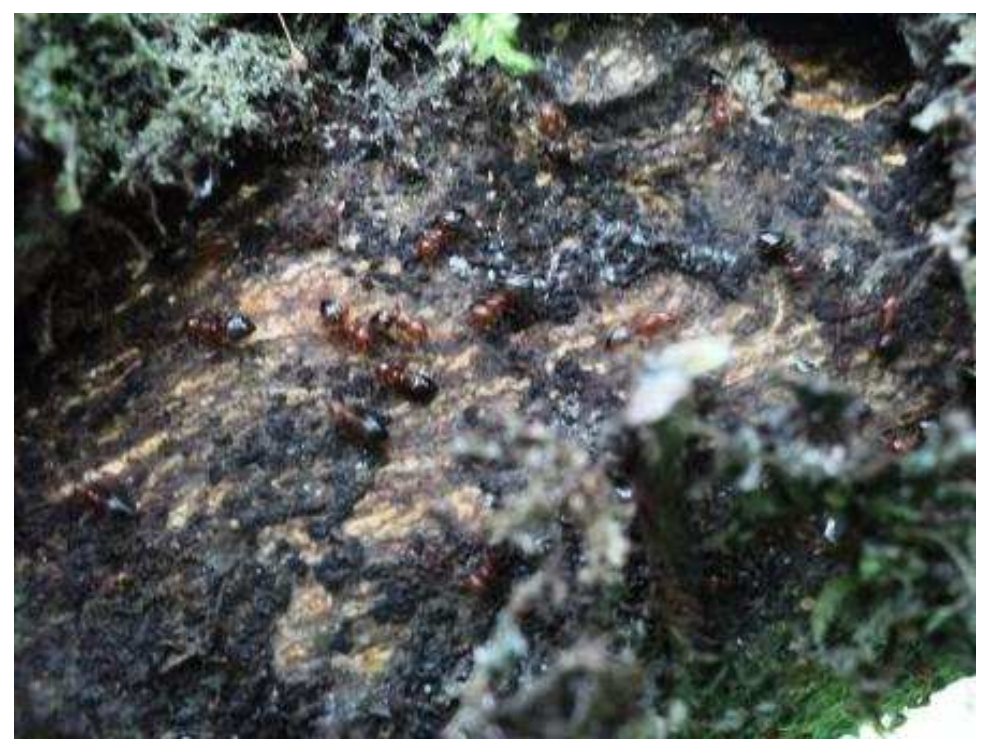

Figure 4 : Crematogaster decacrema. 


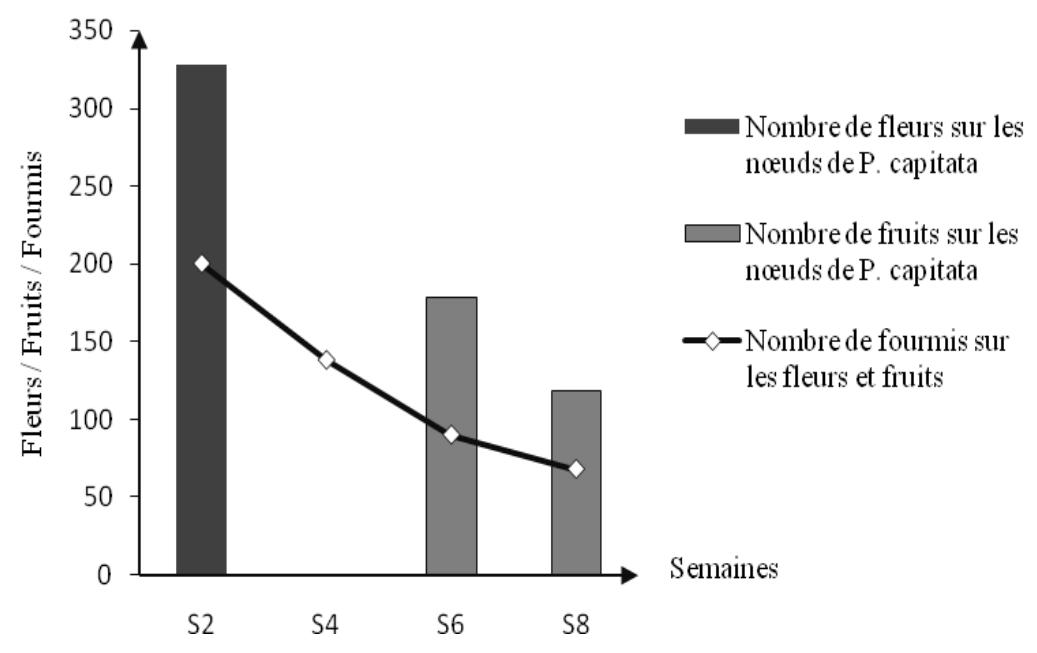

Figure 5: Variation des fourmis pendant la floraison et la fructification de Phragmanthera capitata.

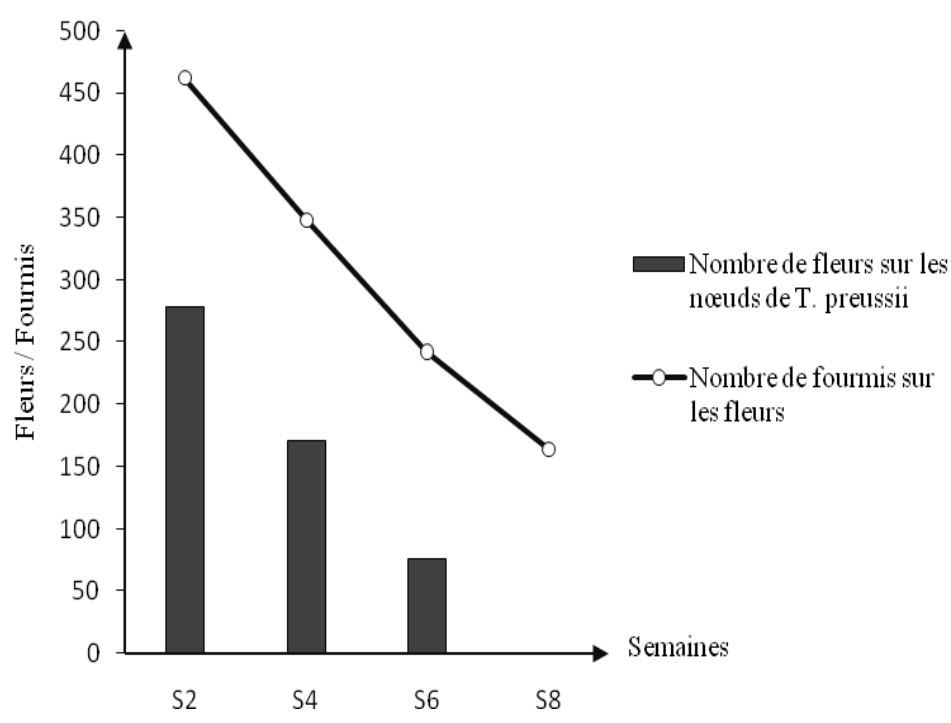

Figure 6 : Variation des fourmis pendant la floraison de Tapinathus preussii. 


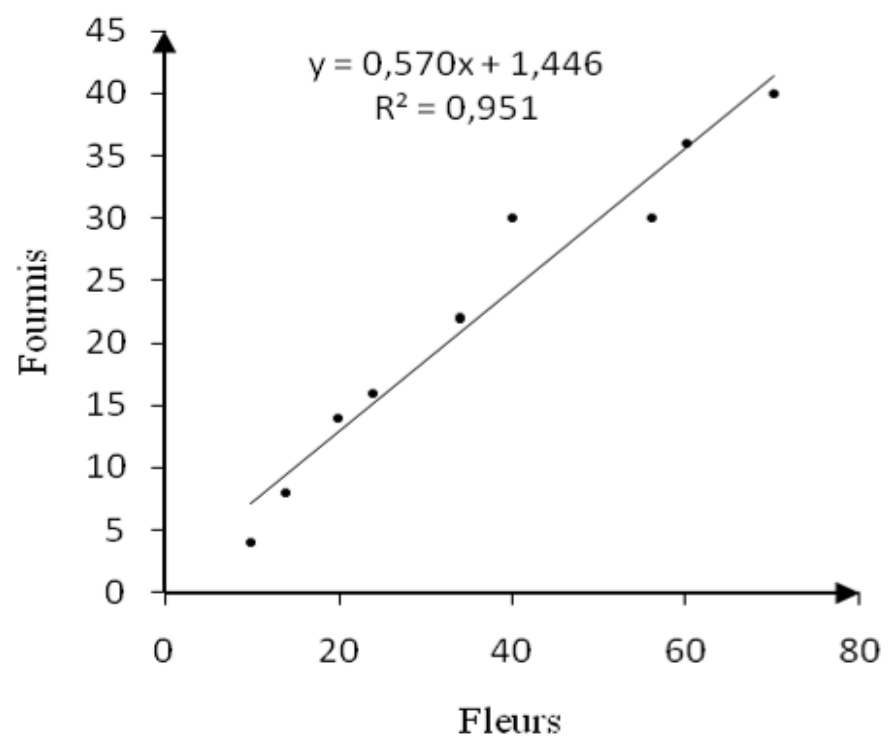

Figure 7 : Corrélation entre les fourmis et les fleurs de Phragmanthera capitata.

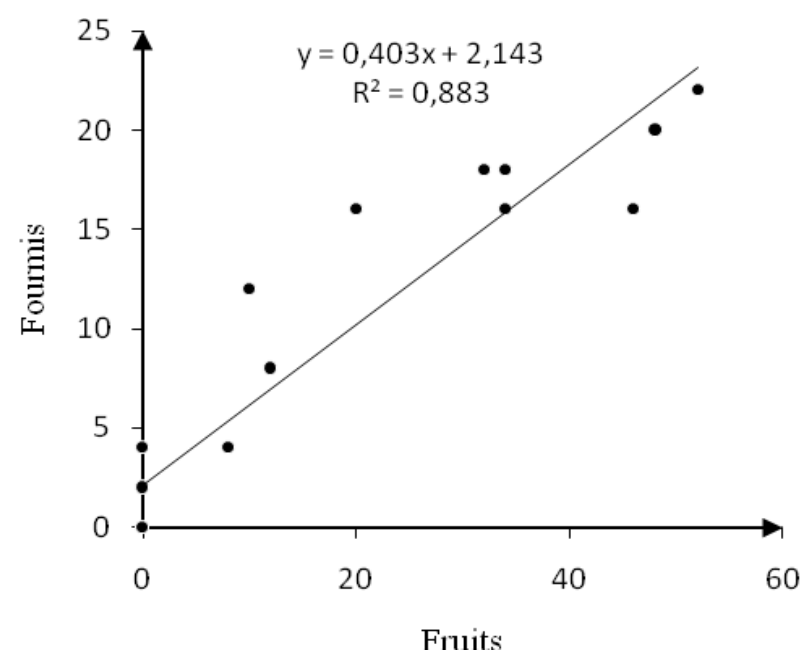

Figure 8 : Corrélation entre les fourmis et les fruits de Phragmanthera capitata. 


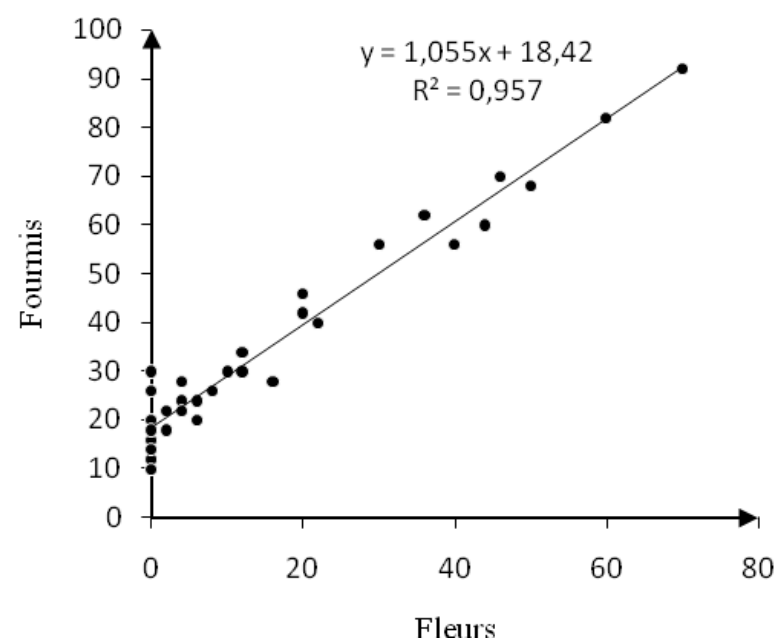

Figure 9 : Corrélation entre les fourmis et les fleurs de Tapinanthus preussii.

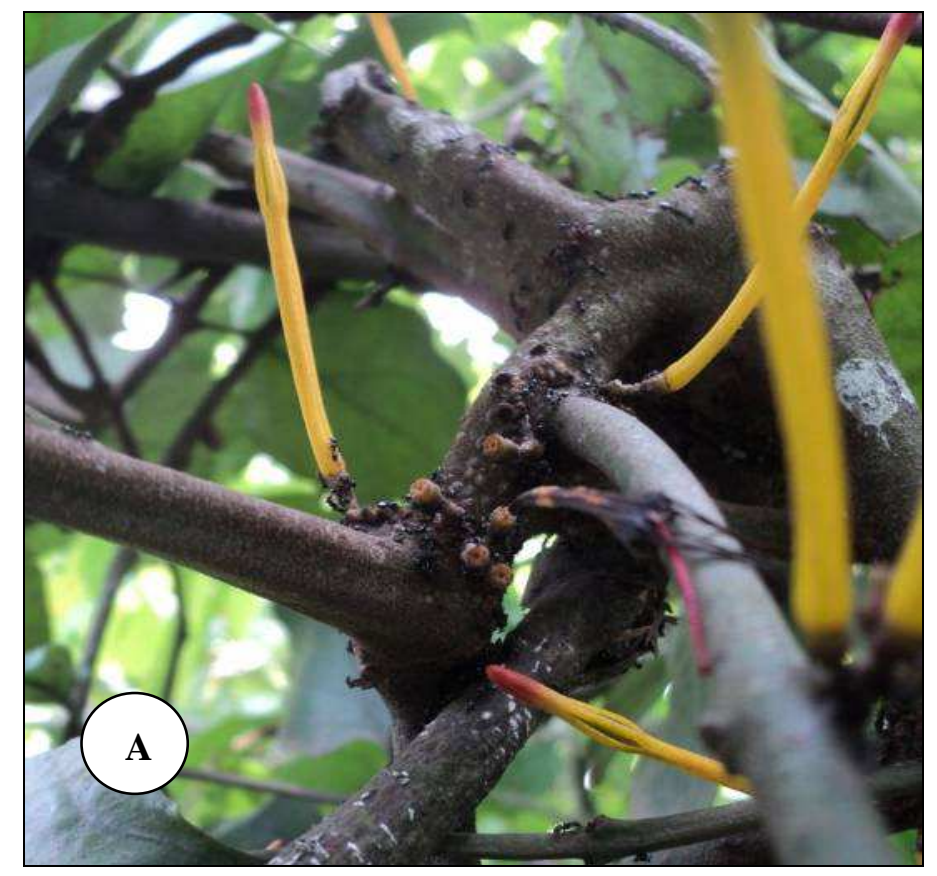


C. F.LADOH YEMEDA et al. / Int. J. Biol. Chem. Sci. 7(3): 924-937, 2013
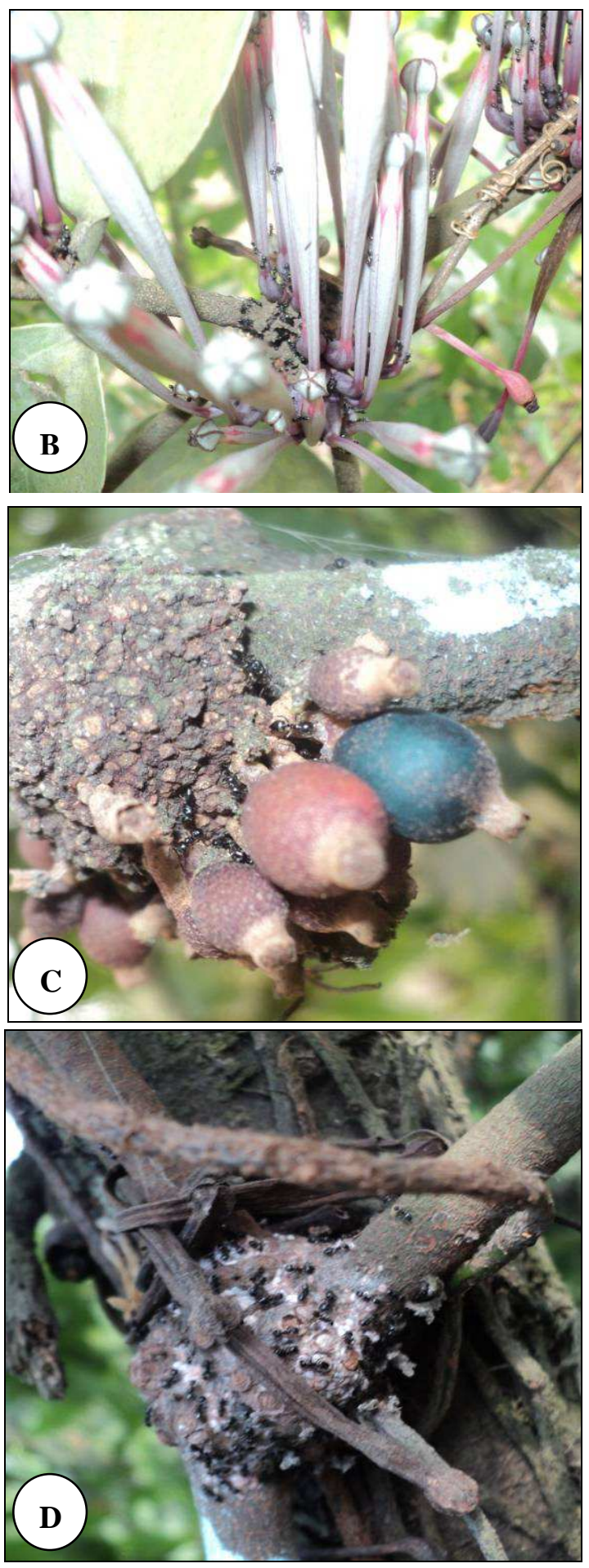

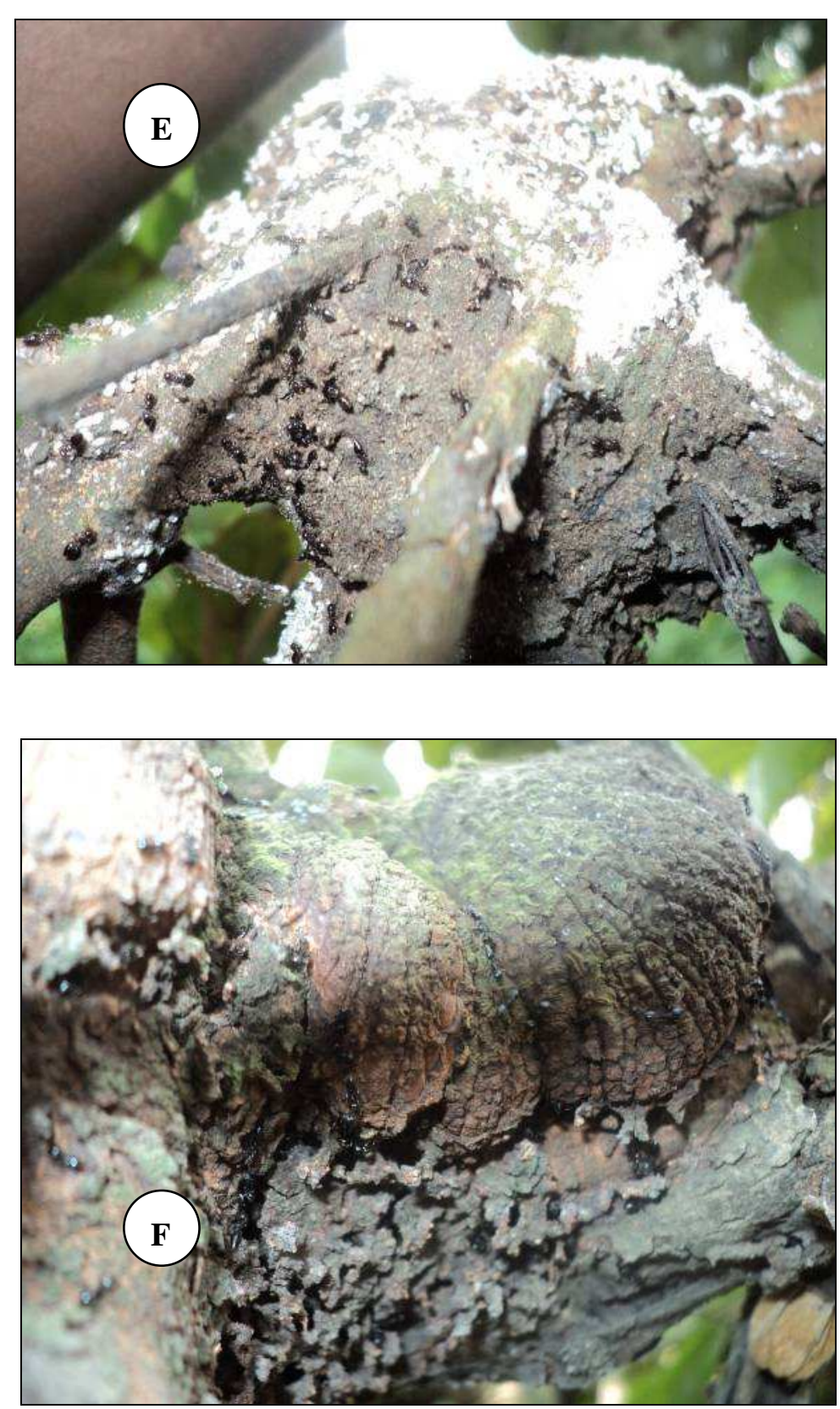

Figure 10 : Activité fourragère des fourmis sur les Loranthaceae. Fourmis à la base (A): des fleurs de Phragmanthera capitata; (B) : des fleurs de Tapinanthus preussii ; (C) : des fruits de P. capitata; (D) : Fourmis sur un nœud de $P$. capitata après la chute des fleurs ; (E) : Fourmis sur un suçoir de $P$. capitata ; (F) : Galéries de fourmis dans un suçoir de $T$. preussii. 


\section{DISCUSSION}

Les sous-familles de fourmis (Formicinae, Ponerinae et Myrmicinae) présentent sur les individus de Theobroma cacao du verger de la chefferie sont similaires à celles obtenues par Mony et al. (2009). Deux sous-familles (Formicinae et Myrmicinae) sont majoritairement représentées par des espèces dominantes. Ces dernières sont caractérisées par les sociétés très populeuses et une agressivité intra et interspécifiques. Le type de ressource alimentaire disponible est aussi l'un des critères à prendre en compte chez ces espèces, en particulier le miellat des Hémiptères riche en sucre, qui permet de pallier aux dépenses énergétiques de la colonie (Blüthgen et al., 2000, 2004 ; Dejean et al., 2000 ; Davidson et al., 2003, 2004; Davidson, 2005). La présence des dominantes sur un arbre exclut ou tolère peu de fourmis, d'où la faible diversité observée sur Theobroma cacao.

L'alimentation des fourmis arboricoles est en grande partie liquide comparée à celles des fourmis terricoles (McKey et al., 1999). Ceci est facilité dans la sous-famille des Myrmicinae, par la présence d'un proventricule rigide et passif (Davidson, 1997; Davidson et al., 2004), structure ayant normalement pour rôle de réguler musculairement le débit d'aliments du jabot social vers l'intestin médian. Bien que cette alimentation soit essentiellement liquide par le biais de la trophobiose sur les Hémiptères, elle associe aussi la capture des proies. Les travaux de terrain menés par Philpott et Armbrecht (2006) et ceux de Davidson et al. (2003) sur l'analyse de l'azote l'ont bien démontrés. Chez le genre Crematogaster, les zones de fourragement sont fixes (feuilles, fleurs, fruits, branches et suçoirs) et proches des nids creusés dans les suçoirs. En contrepartie, elles assurent la défense de cette Loranthaceae contre les herbivores et/ou les plantes compétitrices environnantes. Il s'agit d'un cas classique de relation Plantes-fourmis. La particularité dans cette étude est que ces nids s'observent uniquement chez $T$. preussii quand bien même cette dernière est associée à $P$. capitata sur un même individu hôte parasité de T. cacao. Pourtant Mony et al. (2009), Dibong et al. (2010) signalent la présence de nids sur les suçoirs de $P$. capitata lorsqu'elle parasite seule d'autres arbres du verger de la chefferie. Nous pourrions penser à une forme de compétition ou de préférence dans l'occupation des sites par les fourmis quand $T$. preussii et $P$. capitata sont mis ensembles sur le même support. Mieux encore, T. preussii serait plus attractif pour les fourmis que $P$. capitata.

Le genre Crematogaster tolère les Hémiptères abondants sur les feuilles grasses de Loranthaceae, souvent exploitées pour leur miellat. Selon Blüthgen et al. (2000), Hossaert-Mckey et al., (2001), Blüthgen et al. (2004), la trophobiose avec les Hémiptères est un caractère primordial des fourmis arboricoles dominantes. Les fourmis du genre Crematogaster peuvent par association aux Hémiptères, limiter la propagation des Loranthaceae sur les espèces hôtes contribuant ainsi à leur lutte biologique. Plutôt que d'éliminer toute l'entomofaune par pulvérisation, il serait bénéfique de favoriser l'implantation ou le maintien de certaines populations de fourmis au dépend d'autres, c'est-à-dire de manipuler la mosaïque en favorisant les espèces dominantes qui ne posent pas de problème agronomique (Hölldobler et Wilson, 1990; Mercier, 1999; Dejean et Corbara, 2002).

Pour qu'une fourmi soit intéressante dans la lutte biologique contre les Loranthaceae, elle doit être une excellente tolérante d'agents vecteurs de maladies phytopathologiques et avoir une action indirecte augmentant la dispersion des spores 
de champignons pathogènes (Dejean et al., 1991). Une telle fourmi provoque également des dégâts directs aux Loranthaceae parmi lesquels la défoliation, la récolte des graines et la destruction des suçoirs.

\section{Conclusion}

L'inventaire floristique effectué dans le verger de la chefferie de Ndogbong donne un taux de parasitisme de $59,18 \%$. P. capitata a été la Loranthaceae la plus représentée avec un pourcentage de $97,4 \%$. Toutes les espèces ligneuses à l'exception de Mangifera indica étaient parasitées par les Loranthaceae.

Le suivi de la phénologie des Loranthaceae montrent que les fourmis participent à la chute des fleurs et des fruits limitant ainsi la propagation des Loranthaceae. Deux espèces de fourmis arboricoles dominantes ont été recensées pendant la floraison et la fructification : Crematogaster decacrema et C. oxygyne.

Les fourmis ont contribué significativement à la faible production florifère et fruitière des Loranthaceae. Sachant que la véritable lutte contre les plantes parasites est celle qui empêche la propagation des graines, il serait judicieux de faire des études plus étendues concernant les fourmis arboricoles dominantes afin de voir la possibilité de limiter la prolifération des Loranthaceae dans les vergers, les jardins de cases et dans les plantations.

\section{REFERENCES}

Balle S. 1986. Loranthacées, Flore du Cameroun ( vol. 23), Satabié B, Leroy JF (eds). D.G.R.S.T.: Yaoundé, Cameroun ; $82 \mathrm{p}$.

Beattie AJ, Hughes L. 2002. Ant-plant interactions. In Plant-Animal Interactions. An Evolutionary Approach, Herrera CM, Pellmyr O (eds). Blackwell Publishing: Oxford, UK; 211-235.
Blüthgen N, Stork NE, Fiedler K. 2004. Bottom up control and co-occurrence in complex communities: honeydew and nectar determine a rainforest ant mosaic. Oikos, 106: 344-358.

Blüthgen N, Verhaagh M, Goitia W, Jaffé K, Morawetz W, Barthloot W. 2000. How plant shape the ant community in the Amazonian rainforest canopy: the key role of extrafloral nectarines and hemipteran honeydew? Oecologia, 125: 229-240.

Boussim IJ, Sallé G, Guinko S. 1993. Tapinanthus parasite du karité au Burkina Faso ; 2è partie: Phénologie, biologie et dégâts. Bois et Forêts des Tropiques, 38: 53-65.

Davidson DW. 2005. Ecological stoichiometry of ants in a new World rain forest. Oecologia, 142: 221-231.

Davidson DW. 1997. The role of resource imbalance in the evolutionary ecology of tropical arboreal ants. Biological Journal of the Linnean Society, 61: 153-181.

Davidson DW, Cook SC, Snelling RR. 2004. Liquid-feeding performances of ants (Formicidae): Ecological and Evolutionary implications. Oecologia, 139: 255-266.

Davidson DW, Cook SC, Snelling RR, Chua TH. 2003. Explaining the abundance of ants in lowland tropical forest canopies. Science, 300: 969-972.

Dejean A, Corbara B. 2002. Review on mosaics of dominant ants in rainforests and plantations. In Arthropods of Tropical Forests. Spatio-temporal Dynamics and Resource Use in the Canopy, Basset Y, Novotny V, Miller SE, Kitching RL (eds.). Cambridge Univ. Press: Cambridge, UK.

Dejean A, Corbara B, Orivel J, Leponce M. 2007. Rainforest Canopy Ants: The implications of territoriality and 
Predatory Behavior. Functional Ecosystems and Communities, Global Science Books, 1: 105-120.

Dejean A, Giberneau M. 2000. A rainforest ant mosaic: the edge effect. Sociobiology, 35: $385-401$.

Dejean A, Nkongmeneck B, Corbara B, Djieto-Lordon C. 1991. Impact des fourmis arboricoles sur une pullulation d'Achaea catocaloides (Lepidoptera, Noctuidae) dans les plantations de cacaoyers du Cameroun, et étude de leurs Homoptères associés. Acta Oecologia, 12: 471-488.

Dibong SD, Ndiang Zenabou, Mony R, Boussim Issaka Joseph, Amougou Akoa. 2010. A parasitic study of Phragmantera capitata (Sprengel) S. Balle (Loranthaceae) in the anthropic environments: The case of the Ndogbong chieftain's compound orchard (Douala, Cameroon). African Journal Agricultural Research, 5(15): 2051 - 2055.

Din N, Saenger P, Priso JR, Dibong SD, Amougou A. 2008. Logging activities in mangrove forests: A case study of Douala Cameroon. African J. Environ. Sci. Technol., 2(2): 22-30.

Heil M, Mckey D. 2003. Protective ant-plant interactions as model systems in ecological and evolutionary research. Annual Review of Ecology and Systematics, 34: 425-453.

Hölldobler B, Wilson EO. 1990. The Ants. Harvard University Press: Cambridge, Mass; 732 p.

Hossaert-Mckey M, Orivel J, Laberie E, Pascal L, Delabie JHC, Dejean A. 2001. Differential associations with ants of three co-occuring extrafloral nectarbearing plants. Ecoscience, 8: 325-335.

Gray JS, McIntyre AD, Stirn J. 1992. Manuel des méthodes de recherche sur l'environnement aquatique. Onzième partie. Evaluation biologique de la pollution marine, eu égard en particulier au benthos. FAO Document Technique sur les Pêches, 324: 53-54.

McKey D, Gaume L, Dalecky A. 1999. Les symbioses entre plantes et fourmis arboricoles. L'Année Biologique, 38: 169194.

Mercier JL. 1999. Territorialité et agressivité intra et inter-spécifique dans les mosaïques de fourmis arboricoles. L'Année Biologique, 38: 149-168.

Mony R, Ondoua JM, Dibong SD, Boussim IJ, Amougou A, Bilong Bilong. 2009. Myrmécofaune arboricole associée aux couples Phragmanthera capitata (Sprengel) S. Balle/hôte au verger de la chefferie de Ndogbong (Douala, Cameroun). International Journal Biological and Chemical Sciences, 3(6): 1346-1356.

Passera L, Aron S. 2005. Les Fourmis: Comportement, Organisation Sociale, Evolution. Les Presses Scientifiques du CNRC, Otawa, Canada, 480p.

Philpott S, Armbrecht I. 2006. Biodiversity in tropical agroforests and the ecological role of ants and ant diversity in predatory function. Ecological Entomology, 31: 369-377.

Polhill R, Wiens DW. 1998. Misteltoes of Africa. Kew Ed.: Kew; 370.

Sallé G, Tuquet C, Raynal-Roques A. 1998. Biologie des Phanérogames parasites. $C$. R. Sociobiologie, 192: 9-36.

Sonké B, Kenfack D, Tindo M. 2000. Parasitisme de l'avocatier (Persea americana Mill, Lauraceae) par les Loranthacées dans la région de Yaoundé (Cameroun). Fruits, 55: 325- 331. 